\title{
APPROXIMATE CONDITIONS FOR THE OFF-AXIS TRIPLICATION IN TRANSVERSELY ISOTROPIC MEDIA
}

\author{
V. VAVRYČUK \\ Geophysical Institute, Academy of Sciences of the Czech Republic, Boční II/1401, 14131 Praha 4, \\ Czech Republic (vv@ig.cas.cz) \\ Received: 22 April 2003; Revised: 31 July 2003; Accepted: 14 August 2003
}

\begin{abstract}
The presented approximate formulas yield a critical value of anisotropy parameter $\sigma$, for which an incipient off-axis $S V$-wave triplication occurs in transversely isotropic media. The formulas are simple but approximate the exact solution with a high accuracy. The best results are obtained using the third-order approximation, which yields accuracy at least 30 times higher than the formulas presented by Thomsen and Dellinger (2003). The formula works safely for parameters $\kappa=a_{33} / a_{44}>2$ and $0.2>$ $\varepsilon=\left(a_{11}-a_{33}\right) / 2 a_{33}>-0.2$, and yields critical values of $\sigma$ from 0.1 to 0.7. Outside this interval, it is recommended to use an exact solution.
\end{abstract}

\section{INTRODUCTION}

In homogeneous transverse isotropy, the triplications can occur for the SV wave only and can be classified into four different types (Musgrave, 1970; Payton, 1983): (1) offaxis triplication, (2) on-axis triplication near the symmetry plane, (3) on-axis triplication near the symmetry axis, and (4) double on-axis triplication (see Fig. 1). The triplications are delimited by two cuspidal edge lines on the wave surface. These lines (also called caustics) produce energy focusing (Kravtsov and Orlov, 1990; Wolfe, 1998) and phase shifting of signals (Bakker, 1998; Červený, 2001). The cuspidal lines (shown as cuspidal points in Fig. 2) correspond to two lines of inflection points on the slowness surface characterized by the zero Gaussian curvature (see Fig. 3). The lines of inflection points are also called parabolic lines (Vavryčuk, 2003a). If the two parabolic lines are not separated but coalesce into one, we call this 'incipient' triplication (Helbig, 1994, p. 231). Such media represent the borderline between media with and without a triplication.

The conditions under which the triplications occur in transverse isotropy are well known (Helbig, 1958; Musgrave, 1970; Payton, 1983; Dellinger, 1991). They are elementary except for the off-axis triplication, which is algebraically more involved. For this case, several authors proposed simpler but approximate triplication conditions (McCurdy, 1974; Musgrave, 1979; Musgrave and Payton, 1984; Alshits and Chadwick, 1997; Thomsen and Dellinger, 2003). Among these conditions, the condition proposed by Thomsen and Dellinger (2003) is particularly interesting, because it is far simpler than the other approximations, and provides an insight into which parameters control the off-axis 
triplication. The validity of the formula is, however, restricted to a limited interval of anisotropy parameters. Outside this interval, the accuracy of the formula is rather low. The aim of this study is to present an approximation which is more accurate and applicable to a broader interval of anisotropy parameters.

a)

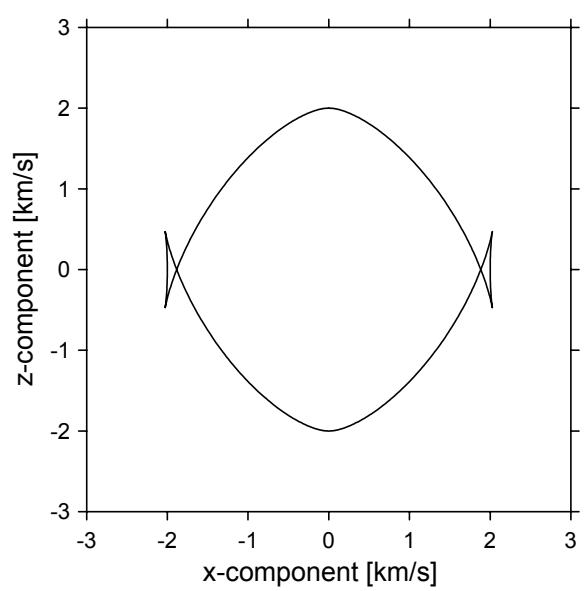

c)

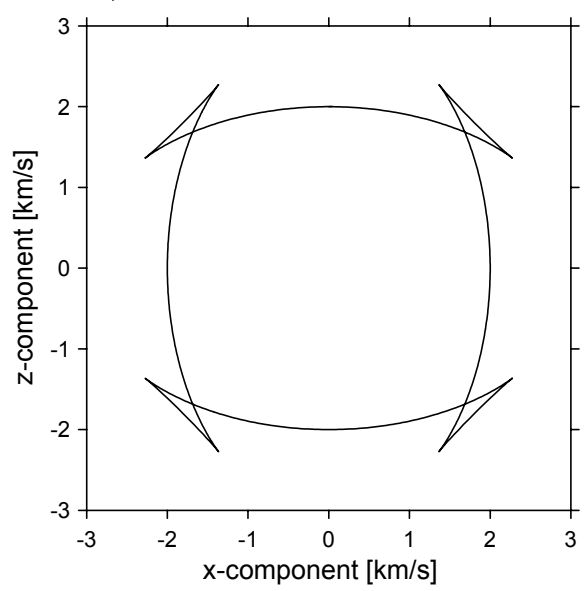

b)

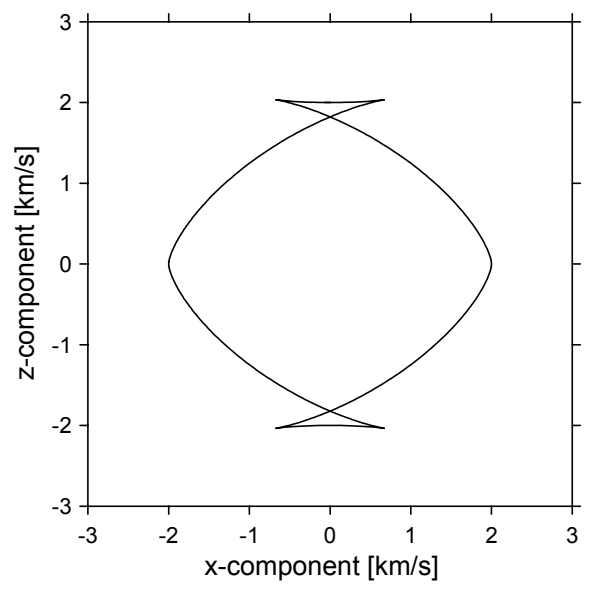

d)

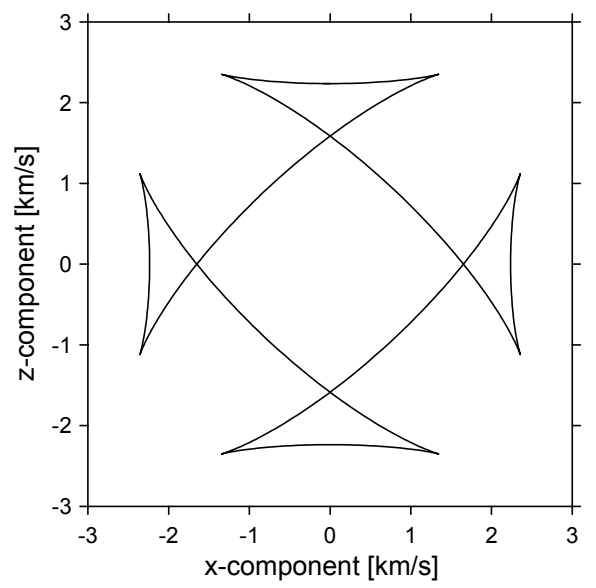

Fig. 1. Types of triplications in transverse isotropy. Vertical sections of wave surfaces are shown for (a) on-axis triplication near the symmetry plane, (b) on-axis triplication near the symmetry axis, (c) off-axis triplication, and (d) double on-axis triplication. 
a)

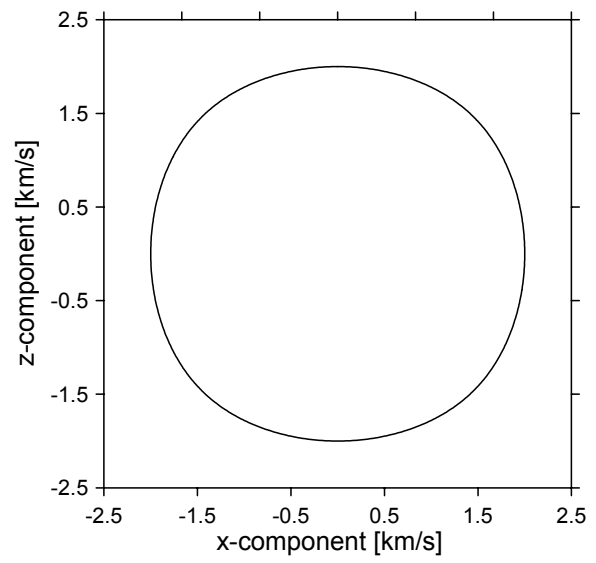

c)

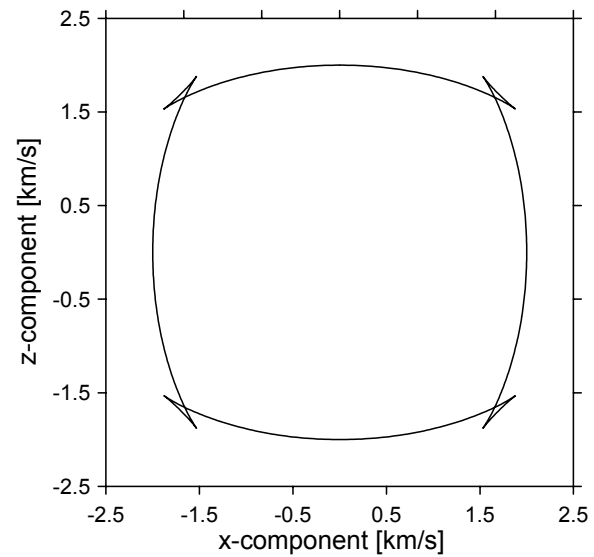

b)

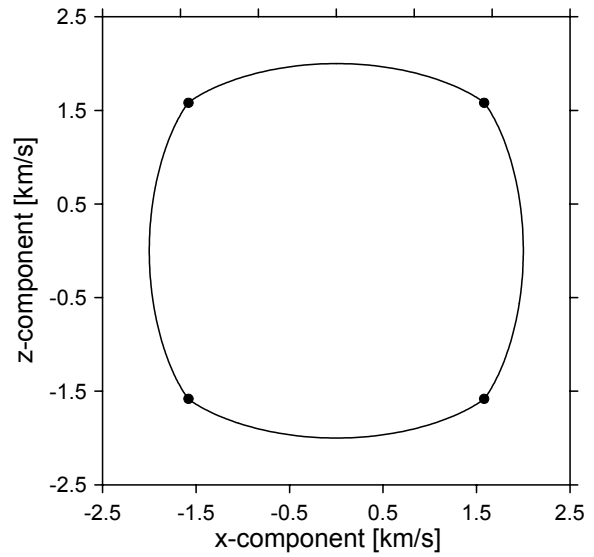

d)

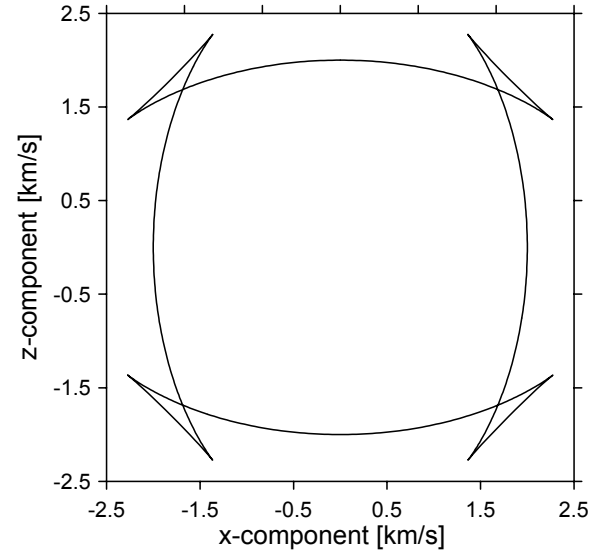

Fig. 2. Off-axis triplications. (a) No triplication, (b) incipient triplication, (c) small triplication, (d) distinct triplication. Elastic parameters are (in $\mathrm{km}^{2} / \mathrm{s}^{2}$ ): $a_{11}=a_{22}=a_{33}=10, a_{44}=a_{55}=4$, $a_{66}=5, a_{12}=a_{11}-2 a_{66}=0$, and (a) $a_{13}=1.5$, (b) $a_{13}=0$, (c) $a_{13}=-1.5$, (d) $a_{13}=-3$. The dots in plot (b) mark the directions of the incipient triplication. 
a)

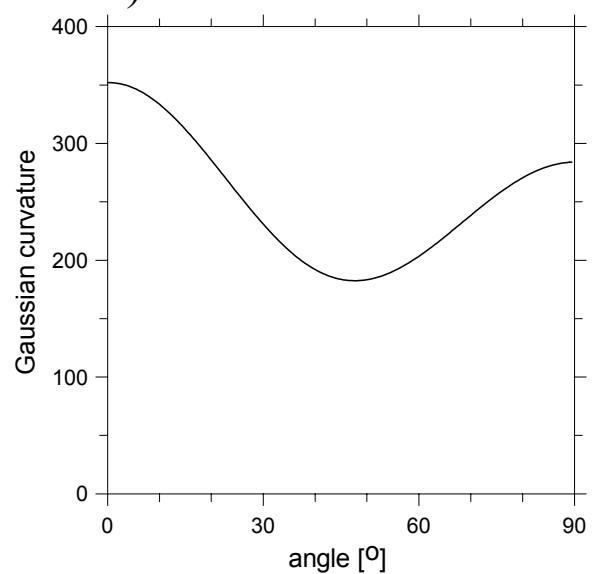

c)

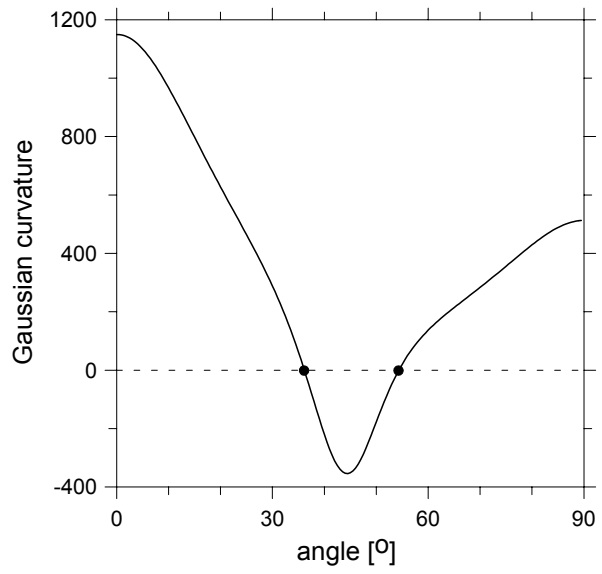

b)

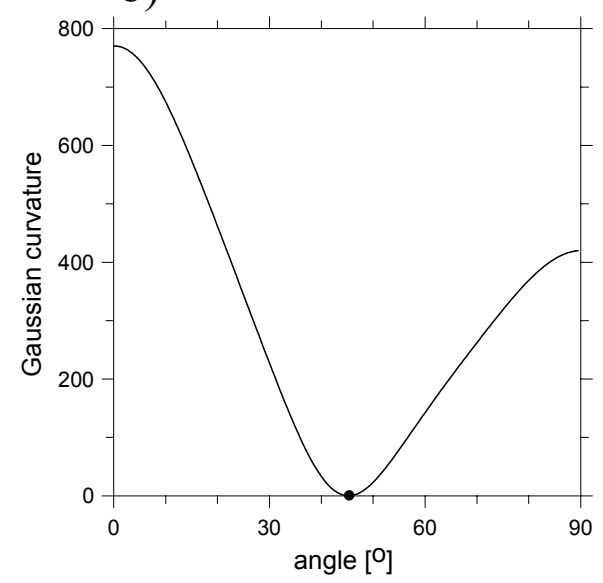

d)

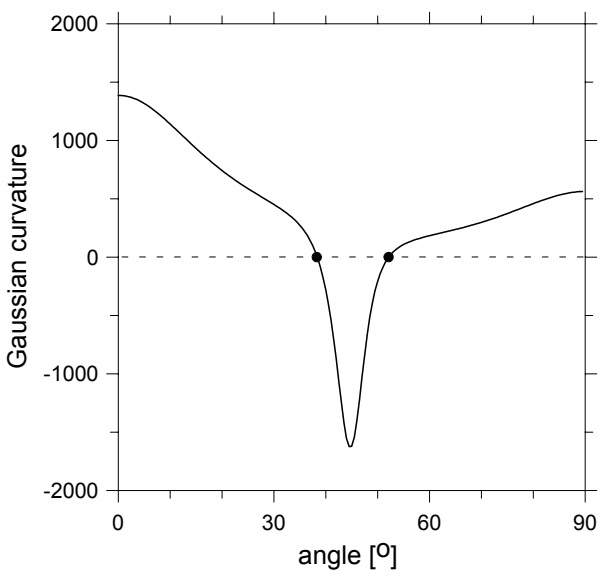

Fig. 3. The Gaussian curvature. (a) No triplication, (b) incipient triplication, (c) small triplication, (d) distinct triplication. The angle measures the deviation of the slowness vector from the symmetry axis. The dots mark inflection points on the slowness surface. For elastic parameters of the media, see the caption of Fig. 2. 


\section{Approximate Conditions for the Off-Axis Triplication ...}

\section{EXACT TRIPLICATION CONDITION}

In this analysis we shall consider transversely isotropic media that satisfy the stability conditions (Backus, 1962, Eq. 20; Helbig, 1994, Eqs 5.3 and 5.23),

$$
a_{33}>0, \quad a_{44}>0, \quad a_{66}>0, a_{11}-a_{66}>0, a_{33}\left(a_{11}-a_{66}\right)>a_{13}^{2},
$$

the condition for 'normal polarization' (Helbig and Schoenberg, 1987),

$$
a_{13}+a_{44}>0
$$

and the 'separation' conditions, which prevent the $P$ and $S V$ slowness or phase-velocity surfaces from intersecting,

$$
a_{11}-a_{44}>0, \quad a_{33}-a_{44}>0,
$$

where $a_{k l}$ are the density normalized elastic parameters in Voigt notation. The stability conditions are necessary for the medium to be physically realizable. The normal polarization and separation conditions are met by overwhelming majority of real materials. For analysis under less restrictive conditions than (2) and (3), see Payton (1983) and Alshits and Chadwick (1997).

The condition for the off-axis triplication is expressed as follows (Dellinger, 1991, Eq. 2.19; Thomsen and Dellinger, 2003, Eq. 9):

$$
\begin{aligned}
\left(a_{13}+a_{44}\right)^{2} & -3 a_{44}^{2}+a_{44}\left(a_{33}+a_{11}\right)-3 a_{11} a_{33} \\
& +2 \sqrt{\left(a_{33}-a_{44}\right)\left(a_{11}-a_{44}\right)} \frac{a_{11} a_{33}-a_{44}^{2}}{a_{13}+a_{44}} \geq 0,
\end{aligned}
$$

where the equality sign stands for the incipient triplication that occurs at the slowness angle $\theta_{i}$ defined by the following equations:

$$
\sin ^{2} \theta_{i}=\frac{a_{33}-a_{44}}{a_{11}+a_{33}-2 a_{44}}, \cos ^{2} \theta_{i}=\frac{a_{11}-a_{44}}{a_{11}+a_{33}-2 a_{44}} .
$$

Condition (4) can also be expressed using the weak-anisotropy parameters $\sigma$ and $\varepsilon$ or $\sigma$ and $\delta$, which represent alternative parameterizations of transverse isotropy (Thomsen, 1986; Tsvankin and Thomsen, 1994):

$$
\varepsilon=\frac{a_{11}-a_{33}}{2 a_{33}}, \quad \delta=\frac{\left(a_{13}+a_{44}\right)^{2}-\left(a_{33}-a_{44}\right)^{2}}{2 a_{33}\left(a_{33}-a_{44}\right)}, \quad \sigma=\frac{a_{33}}{a_{44}}(\varepsilon-\delta) .
$$

These parameters are frequently used in describing transverse isotropy. They become zero in isotropy and can serve as a measure of strength of transverse isotropy. Therefore, expressing the triplication condition using these parameters, we can better understand how strong anisotropy must be to generate triplications (Vavryčuk, 2003b). Among these parameters, the crucial role is played by the parameter $\sigma$ in the triplication condition, 
because it controls the variation of the phase velocity of the $S V$ wave in the weakanisotropy approximation

$$
c_{S V}^{2}=a_{44}\left(1+2 \sigma \sin ^{2} \theta \cos ^{2} \theta\right),
$$

where $\theta$ is the slowness angle measured from the symmetry axis.

The condition for the off-axis triplication (4) expressed in terms of anisotropy parameters yields the following cubic equation for the critical value $\sigma_{c}$ of the weakanisotropy parameter $\sigma$ at which an incipient triplication occurs

$$
\sigma_{c}^{3}+A \sigma_{c}^{2}+B \sigma_{c}+C=0,
$$

where

$$
\begin{aligned}
& A=\frac{1}{\kappa-1}\left[\frac{3}{2} \kappa^{2}(2 \varepsilon+1)+\kappa(\varepsilon+1)+\frac{3}{2}\right], \\
& B=2 \frac{\kappa}{(\kappa-1)^{2}}(\varepsilon+1)\left[\kappa^{2}(2 \varepsilon+1)+1\right] \\
& C=-2 \frac{\kappa^{2}}{(\kappa-1)^{2}}\left[\kappa(2 \varepsilon+1)^{2}-2 \varepsilon-1\right],
\end{aligned}
$$

and

$$
\kappa=\frac{a_{33}}{a_{44}} .
$$

The solution of Eq. (8) yields an interval of values of the $\sigma$ parameter the medium triplicates for:

$$
\sigma \geq \sigma_{c},
$$

where

$$
\begin{aligned}
& \sigma_{c}=u+v-\frac{1}{3} \\
& u=\left[-\frac{q}{2}+\sqrt{\left(\frac{q}{2}\right)^{2}+\left(\frac{p}{3}\right)^{3}}\right]^{\frac{1}{3}}, \quad v=\left[-\frac{q}{2}-\sqrt{\left(\frac{q}{2}\right)^{2}+\left(\frac{p}{3}\right)^{3}}\right]^{\frac{1}{3}}, \\
& p=B-\frac{1}{3} A^{2}, \quad q=-\frac{1}{3} A B+\frac{2}{27} A^{3}+C .
\end{aligned}
$$


a)

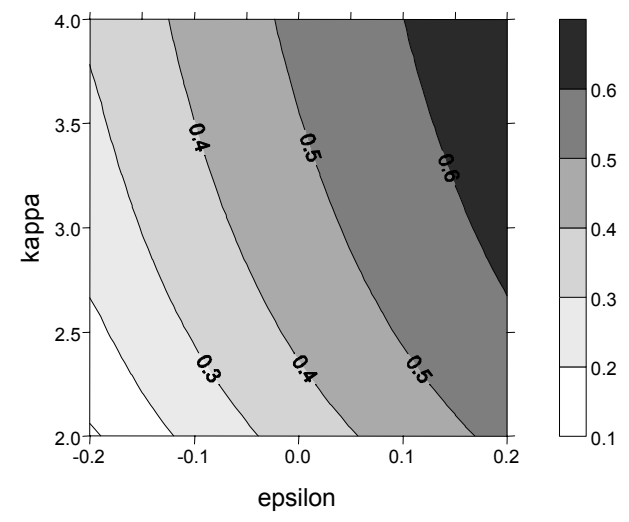

b)

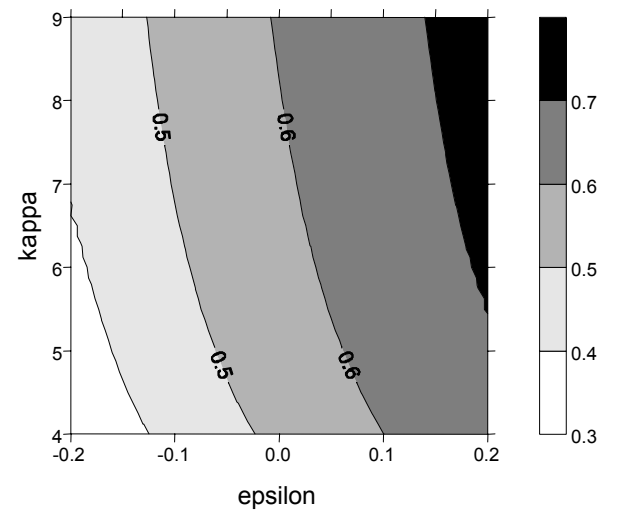

Fig. 4. The critical value $\sigma_{c}$ for the incipient off-axis triplication as a function of parameters $\kappa$ and $\varepsilon$. (a) $2 \leq \kappa \leq 4$, (b) $4 \leq \kappa \leq 9$.

Note that the other two solutions of the cubic equation (8) are spurious, since they do not satisfy conditions (1) or (2).

The behaviour of $\sigma_{c}$ is shown in Fig 4 . The figure shows that $\sigma_{c}$ is significantly influenced by $\kappa$ and $\varepsilon$. Interestingly, low values of $\kappa$ and $\varepsilon$ result in low values of $\sigma_{c}$. For example, if we assume $\kappa=2$ and $\varepsilon=-0.12$, the triplication occurs for $\sigma \geq \sigma_{c}=0.2$. If we define the strength of anisotropy as $\left(c_{\max }-c_{\min }\right) /\left(c_{\max }+c_{\min }\right) \cdot 200 \%$, where $c$ is the phase velocity of the respective wave, then these parameters describe an $S V$ anisotropy of $8 \%$ only. For value $\kappa=3$, corresponding to a $P$-to- $S$ velocity ratio $v_{P} / v_{S}=\sqrt{3}$, and for $\varepsilon$ ranging from -0.2 to 0.2 , we obtain $\sigma_{c}$ in the interval $\sigma_{c} \in\langle 0.24,0.62\rangle$. These values correspond to $S V$ anisotropy in the range of $9 \%$ to $12 \%$.

\section{APPROXIMATE TRIPLICATION CONDITIONS}

The exact solution (11) of the cubic equation (8) is still rather complicated. Therefore, we shall try to simplify it further. The exact solution (11) can be expanded into a power series in parameters $\varepsilon$ and $\kappa^{-1}$. Using only the leading terms of the expansion, we obtain an approximate formula for $\sigma_{c}$ in the form:

$$
\begin{aligned}
& \sigma_{c}=\sigma^{(0)}+\sigma^{(1)}+\sigma^{(2)}+\sigma^{(3)}+\ldots, \\
& \sigma^{(0)}=\frac{2}{3},
\end{aligned}
$$




$$
\begin{aligned}
& \sigma^{(1)}=\frac{2}{3}\left(\varepsilon-\frac{7}{9} \kappa^{-1}\right), \\
& \sigma^{(2)}=\frac{2}{3}\left(-\frac{3}{4} \varepsilon^{2}+\varepsilon \kappa^{-1}-\frac{31}{81} \kappa^{-2}\right), \\
& \sigma^{(3)}=\frac{2}{3}\left(\frac{3}{4} \varepsilon^{3}-\frac{43}{36} \varepsilon^{2} \kappa^{-1}+\frac{49}{81} \varepsilon \kappa^{-2}-\frac{71}{729} \kappa^{-3}\right) .
\end{aligned}
$$

Generally, the higher the order of the expansion is considered, the higher the accuracy of the formula for $\sigma_{c}$ should be obtained. The first two terms of the expansion, $\sigma^{(0)}$ and $\sigma^{(1)}$, were also derived by Thomsen and Dellinger (2003). They obtained the following approximate formulas for $\sigma_{c}$ (Thomsen and Dellinger, 2003, Eq. 19):

$$
\sigma_{c} \cong \frac{2}{3}\left(1+\delta-\frac{1}{9} \kappa^{-1}\right),
$$

or alternatively (Thomsen and Dellinger, 2003, Eq. 23)

$$
\sigma_{c} \cong \frac{2}{3} \frac{1+\varepsilon-\frac{1}{9} \kappa^{-1}}{1+\frac{2}{3} \kappa^{-1}}
$$

Eq. (19) utilizes the $\delta$ parameter instead of $\varepsilon$. As shown later, this lowers the accuracy of this approximation. Eq. (20) is equivalent to the first-order approximation (16) meaning that if expression (20) is expanded into a power series in the small parameters $\varepsilon$ and $\kappa^{-1}$, the corresponding expansion differs from equation (16) by second- or higher-order terms. In the next section, we shall examine accuracy of the derived formulas together with formulas (19) and (20) presented by Thomsen and Dellinger (2003).

\section{TESTS OF ACCURACY}

We test the accuracy of the approximate formulas for $\sigma_{c}$ by calculating the relative errors of the approximations, defined as follows:

$$
e=\frac{\left|\sigma_{c}^{\text {exact }}-\sigma_{c}^{\text {aprox }}\right|}{\sigma_{c}^{\text {exact }}} \cdot 100 \%
$$

The accuracy is examined for the first-, second- and third-order approximations $(14)-(18)$ derived in the previous section and compared with the accuracy of Eqs (19) and (20). We use the following intervals of parameters: $\varepsilon \in\langle-0.2,0.2\rangle$ and $\kappa \in\langle 2,9\rangle$. These intervals are rather broad and should include a majority of geophysically interesting materials (note that the materials with a positive $\varepsilon$ are more frequently observed than those with a negative $\varepsilon$ ). 
a) TD (2003, Eq. 19)

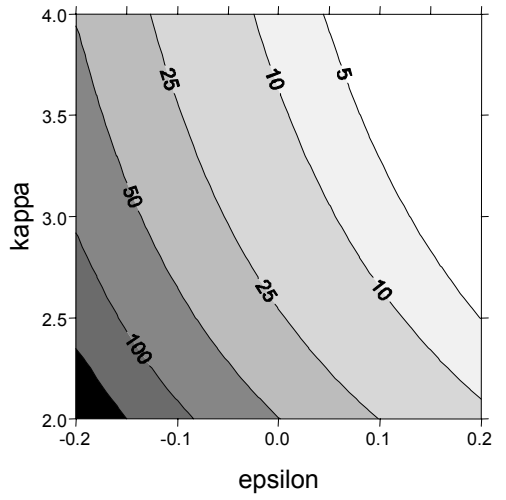

c)
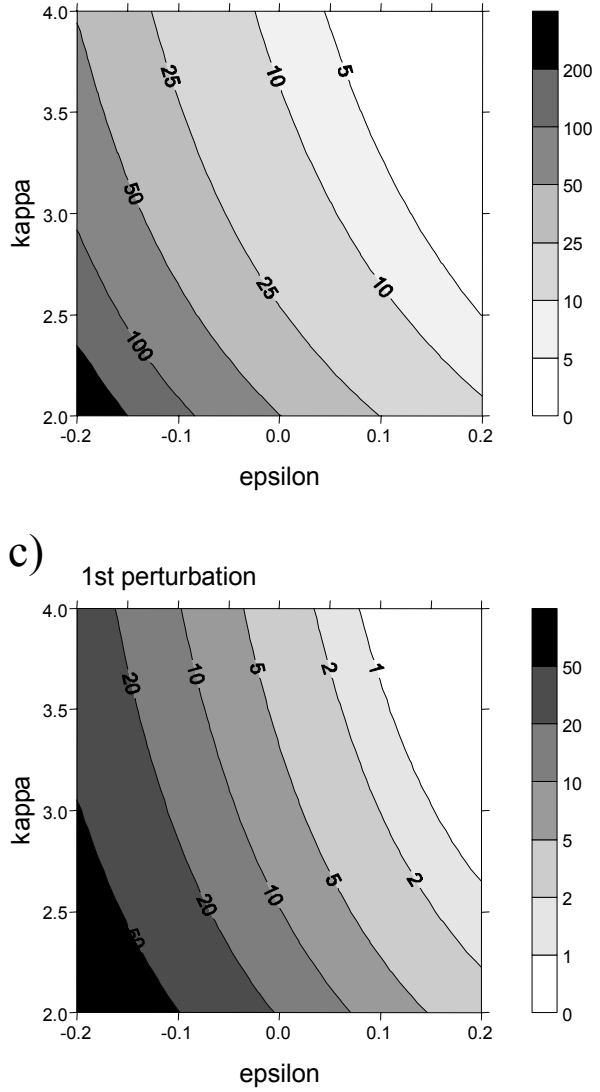

b) TD (2003, Eq. 23)

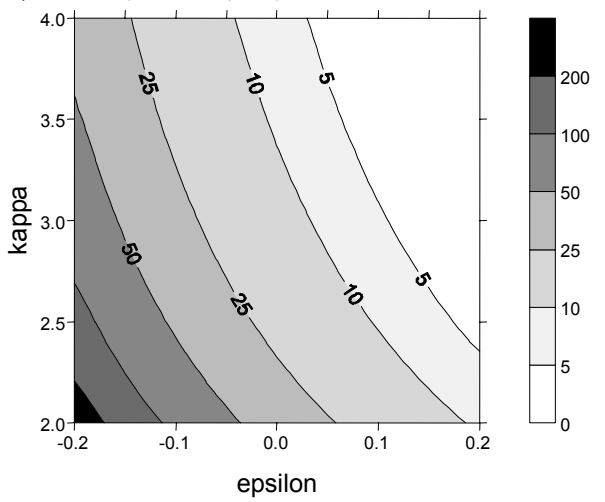

d)

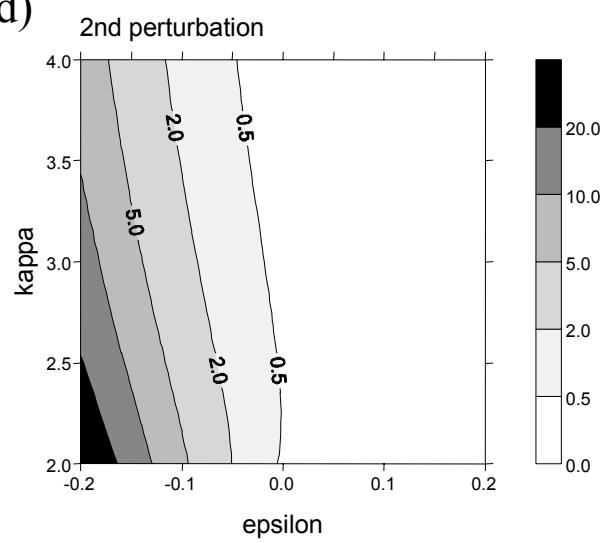

Fig. 5. Relative errors (in per cent) of approximate formulas for $\sigma_{c}, 2 \leq \kappa \leq 4$. (a) Formula by Thomsen and Dellinger (2003, Eq. 19), (b) formula by Thomsen and Dellinger (2003, Eq. 23), (c) first-order approximation (16), (d) second-order approximation (17).

Figure 5 shows the relative errors of the Thomsen and Dellinger (2003) approximations together with the first- and second-order approximations (16) and (17) calculated for $\kappa \in\langle 2,4\rangle$, and Fig. 6 shows the same for $\kappa \in\langle 4,9\rangle$, respectively. Figure 7 shows the relative errors of the third-order approximation (18). The figures indicate that all formulas work better for higher values of $\kappa$ and for positive values of $\varepsilon$. The accuracy is significantly lowered if values of $\kappa$ and $\varepsilon$ decrease. Comparing different approximations, the worst accuracy is obtained using formula (19). For example, this approximation yields an error of about $20 \%$ for values $\kappa=3$ and $\varepsilon=0$. Compared with this approximation, formula (20) is about 1.2 times more accurate and the first-order 
a) TD (2003, Eq. 19)

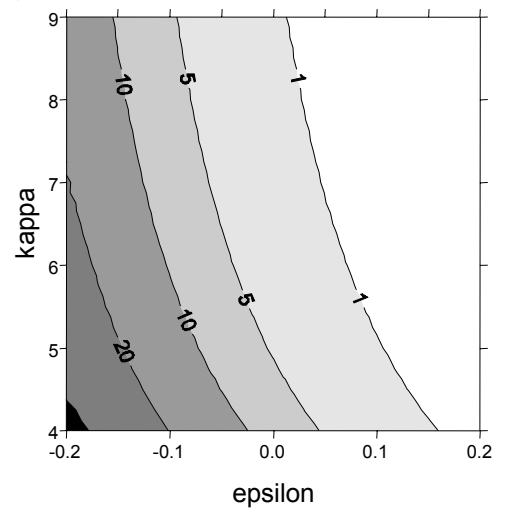

c)

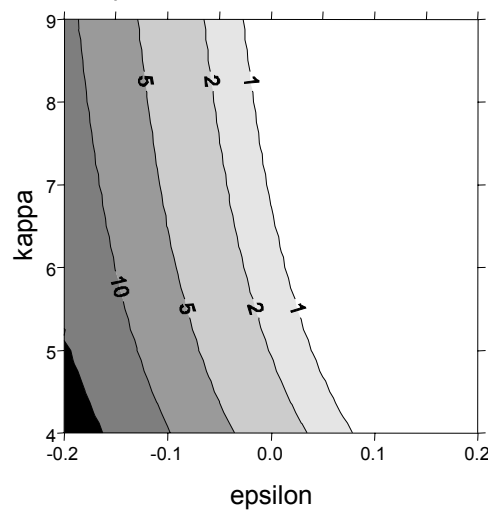

b) TD (2003, Eq. 23)
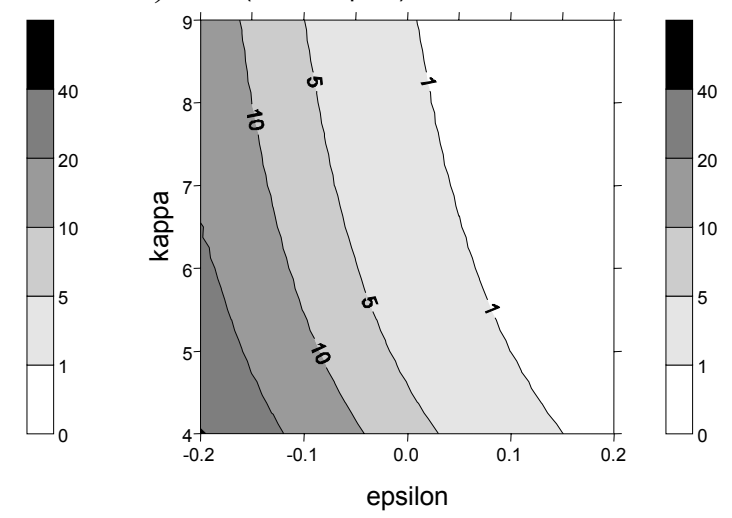

d)

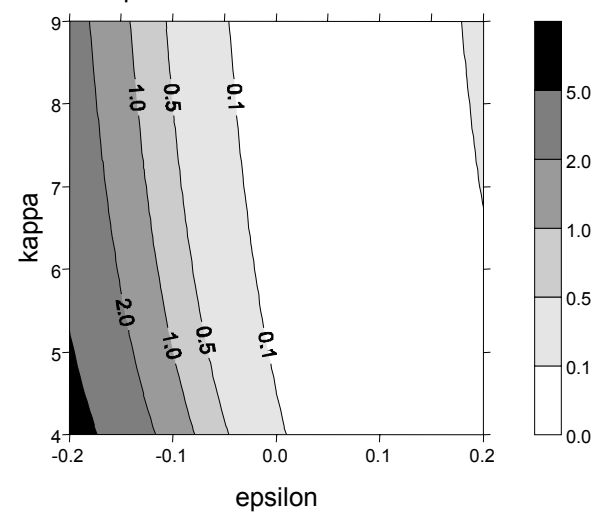

Fig. 6. Relative errors (in per cent) of approximate formulas for $\sigma_{c}, 4 \leq \kappa \leq 9$. (a) Formula by Thomsen and Dellinger (2003, Eq. 19), (b) formula by Thomsen and Dellinger (2003, Eq. 23), (c) first-order approximation (16), (d) second-order approximation (17).

approximation (16) is about two times more accurate. Interestingly, formula (16) is simpler but still more accurate than (20). The second- and third-order approximations (17) and (18) are about 10 and 40 times more accurate than approximation (19), respectively. Note that the comparison of the accuracy of different approximations is very rough and reflects the efficiency of the approximations averaged over the whole considered range of parameters $\kappa$ and $\varepsilon$. Locally, for a specific combination of $\kappa$ and $\varepsilon$, the comparison can yield significantly different values. 
a)

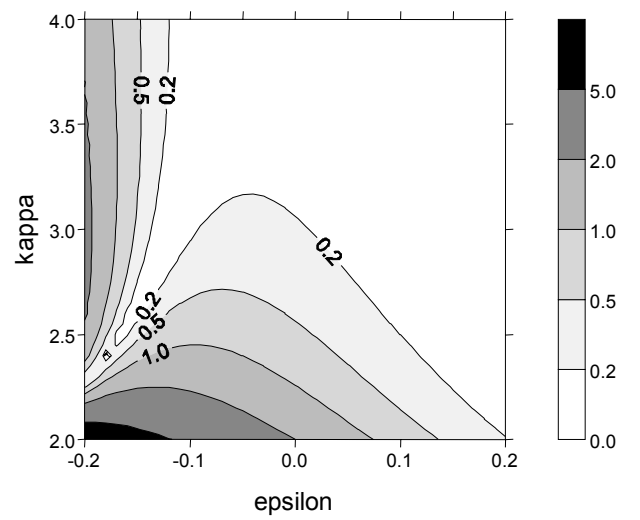

b)

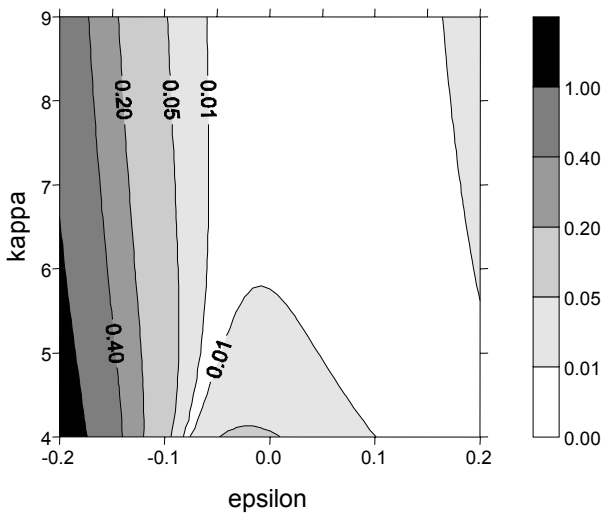

Fig. 7. Relative errors (in per cent) of the third-order approximation (18) for $\sigma_{c}$. (a) $2 \leq \kappa \leq 4$, (b) $4 \leq \kappa \leq 9$.

\section{CONCLUSION}

The proposed approximate formulas yield a critical value $\sigma_{c}$ of the parameter $\sigma$ for which an incipient off-axis $S V$-wave triplication occurs in transversely isotropic media. Although the formulas are simple, they approximate the exact solution with a high accuracy. The best results are obtained by the third-order approximation (14) - (18), which yields an accuracy about 40 and 30 times higher than formulas (19) and (20), respectively. The formula works safely for parameters $\kappa>2$ and $\varepsilon>-0.2$, and is thus applicable to a broad range of TI parameters. Outside this interval, it is recommended to use the exact solution.

The behaviour of $\sigma_{c}$ indicates that triplications do not occur for a fixed strength of anisotropy. The critical strength of anisotropy, i.e. the strength of anisotropy for which the medium triplicates, depends on the parameters $\kappa$ and $\varepsilon$. High values of $\kappa$ and positive $\varepsilon$ produce high values of $\sigma_{c}$, implying that the critical strength of the $S V$-wave anisotropy is rather high. On the contrary, low values of $\kappa$ and negative $\varepsilon$ imply that wave fronts in TI tend to triplicate more easily, meaning that the critical strength of the $S V$-wave anisotropy is low. For a typical value of $\kappa, \kappa=3$, which corresponds to $v_{P} / v_{S}=\sqrt{3}$, and for $\varepsilon$ ranging from -0.2 to 0.2 , parameter $\sigma_{c}$ lies in the interval $\sigma_{c} \in\langle 0.24,0.62\rangle$ (see Fig. 4). These values correspond to $S V$ anisotropy in the range of $9 \%$ to $12 \%$.

Acknowledgments: The author thanks Ivan Pšenčík, Joe Dellinger and an anonymous reviewer for their reviews. The work was supported by the Consortium Project 'Seismic waves in complex 3D structures', and by the Grant Agency of the Academy of Sciences of the Czech Republic, Grant No. A3012309. 


\section{Vavryčuk}

\section{References}

Alshits V.I. and Chadwick P., 1997. Concavities on the zonal slowness section of a transversely isotropic material. Wave Motion, 25, 347-359.

Backus G.E., 1962. Long-wave anisotropy produced by horizontal layering. J. Geophys. Res., 66, $4427-4440$.

Bakker P.M., 1998. Phase shift at caustics along rays in anisotropic media. Geophys. J. Int., 134, $515-518$.

Červený V., 2001. Seismic Ray Theory. Cambridge University Press, Cambridge.

Dellinger J., 1991. Anisotropic Seismic Wave Propagation. PhD Thesis, Stanford University.

Helbig K., 1958. Elastischen wellen in anisotropen medien, Teil I. Gerlands Beiträge zur Geophysik, 67, 177-211.

Helbig K., 1994. Foundations of Anisotropy for Exploration Seismics. Pergamon, N.Y.

Helbig K. and Schoenberg M., 1987. Anomalous polarization of elastic waves in transversely isotropic media. J. Acoust. Soc. Am., 81, 1235-1245.

Kravtsov Yu.A. and Orlov Yu.I., 1990. Geometrical Optics of Inhomogeneous Media. SpringerVerlag, New York.

McCurdy A.K., 1974. Phonon focusing and phonon conduction in hexagonal crystals in the boundary scattering regime. Phys. Rev. B, 9, 466-480.

Musgrave M.J.P., 1970. Crystal Acoustics. Holden-Day, London.

Musgrave M.J.P., 1979. Further criteria for elastic waves in anisotropic media. J. Elasticity, 9, $105-112$.

Musgrave M.J.P and Payton R.G., 1984. Criteria for elastic waves in anisotropic media - a consolidation. J. Elasticity, 14, 269-285.

Payton R.G., 1983. Elastic Wave Propagation in Transversely Isotropic Media. Martinus Nijhoff Publishers, The Hague.

Thomsen L., 1986. Weak elastic anisotropy. Geophysics, 51, 1954-1966.

Thomsen L. and Dellinger J., 2003. On shear-wave triplication in transversely isotropic media. J. Appl. Geophys., 54, 289-296.

Tsvankin I. and Thomsen L., 1994. Nonhyperbolic reflection moveout in anisotropic media. Geophysics, 59, 1290-1304.

Vavryčuk V., 2003a. Parabolic lines and caustics in homogeneous weakly anisotropic solids. Geophys. J. Int., 152, 318-334.

Vavryčuk V., 2003b. Generation of triplications in transversely isotropic media. Phys. Rev. B, 68, in print.

Wolfe J.P., 1998. Imaging Phonons. Acoustic Wave Propagation in Solids. Cambridge University Press, Cambridge. 